\title{
A Study on the Influence of the Nozzle Lead Angle on the Performance of Liquid Metal Electromagnetic Micro-Jetting
}

\author{
Zhiwei Luo ${ }^{1,2, *}$, Gaofeng Zheng ${ }^{3}$ and Lingyun Wang ${ }^{1, *}$ \\ 1 Department of Mechanical and Electrical Engineering, Xiamen University, Xiamen 361005, China \\ 2 School of Mechanical and Automotive Engineering, Xiamen University of Technology, Xiamen 361024, China \\ 3 Department of Instrumental and Electrical Engineering, Xiamen University, Xiamen 361005, China; \\ zheng_gf@xmu.edu.cn \\ * Correspondence: zwluo@xmut.edu.cn (Z.L.); wangly@xmu.edu.cn (L.W.); \\ Tel.: +86-592-629-1386 (Z.L.); +86-592-218-5927 (L.W.)
}

Academic Editors: Andrew J. deMello and Xavier Casadevall i Solvas

Received: 10 November 2016; Accepted: 29 November 2016; Published: 5 December 2016

\begin{abstract}
To improve the jetting performance of liquid metals, an electromagnetic micro-jetting (EMJ) valve that realizes drop-on-demand (DOD) jetting while not involving any valve core or moving parts was designed. The influence of the lead angle of the nozzle on the jetting of liquid metal gallium (Ga) was investigated. It was found that the Lorentz force component parallel to the nozzle that jets the electrified liquid Ga is always larger than its internal friction; thus, jet can be generated with any lead angle but with different kinetic energies. Experimental results show that the mass of the jetting liquid, the jetting distance, the initial velocity of the jet, and the resulting kinetic energy of the jet increase first and then decrease. When the lead angle is $90^{\circ}$, the mass of the jetting liquid and the kinetic energy are at their maximum. When the angle is $80^{\circ}$, the initial velocity achieves its maximum, with a calculated value of $0.042 \mathrm{~m} / \mathrm{s}$. Moreover, very close and comparatively high kinetic energies are obtained at $80^{\circ}$ and $90^{\circ}$, indicating that angles in between this range can produce a preferable performance. This work provides an important theoretical basis for the design of the EMJ valve, and may promote the development and application of micro electromagnetic jetting technology.
\end{abstract}

Keywords: electromagnetic micro-jetting; micro-nozzle; lead angle; liquid metal; Lorentz force

\section{Introduction}

Liquid metal droplet jetting technology was not developed until the 1990s [1]. This technology is highly automatic, environmentally friendly, low-cost, and non-contact [2,3] and can precisely deposit metallic materials such as copper [4], aluminum alloy [5], and tin lead alloy [6] with high efficiency. Its application can be found in many fields, including but not limited to complex metal forming, micro electro mechanical system (MEMS), 3D packaging [7-10], flexible electronics, and microcircuit fast printing [11].

To date, various micro-jetting techniques have been developed for different kinds of metal materials. Driving modes include the pneumatic driving mode, the stress wave driving mode, the electromagnetic force driving mode, and the laser melting mode [12]. According to the jet behavior, they can be classified into a drop-on-demand (DOD) jet and a continuous-ink jet (CIJ). Yuan et al. [13] have developed a pneumatic drive jetting apparatus to print tin lead alloy and aluminum alloy. Later, they integrated ultrasonic supply powder technology into the apparatus to achieve the molding of heterogeneous metal parts [14]. The melted aluminum alloy was jetted in DOD mode by using the compressed gas as the driving source by Cheng et al. [15] and Cao et al. [16] In addition, Chun et al. [17] 
and Orme et al. [18] employed the jet crushing technique to break liquid metals into uniform droplet beams and finally obtained a homogeneous spherical metal powder. However, the above techniques are problematic in terms of the size of the liquid droplet and/or the jetting frequency. The minimum diameter of the liquid droplet for the pneumatic driving mode is normally up to $85 \mu \mathrm{m}$, with a low stable jetting frequency of only $5 \mathrm{~Hz}$. Using the stress wave driving mode, the diameter can be reduced to $63.8 \mu \mathrm{m}$, albeit at a low frequency of $5 \mathrm{~Hz}$. On the other hand, when using the laser melting process, the positioning and the power of the laser needs to be controlled precisely to avoid satellite droplets and to increase the frequency. From the viewpoint of industrial application of liquid metal micro-jetting, the jetting frequency and the stability of the system must be enhanced to print complex patterns, since these two aspects directly affect the efficiency of electronic packaging and 3D printing of metallic materials. For example, some engineering fields require a frequency of more than $150 \mathrm{~Hz}$ for specific applications, which is hardly achievable with existing jetting techniques.

To obtain a more satisfactory jetting process, an electromagnetic micro-jetting (EMJ) technique has been proposed. This technique utilizes the Lorentz force field to drive liquid metals to be jetted, where free electrons inside the electrified liquid metal are subjected to a complex field consisting of the electric field and the magnetic field perpendicular to it, and liquid metals are continuously ejected in a specific direction. This technique is characterized by free of moving parts, noise or vibration, meanwhile achieving high jetting frequency. To further demonstrate its performance, EMJ valves with different nozzle lead angles were designed, and experiments were performed.

\section{Experiment}

In order to achieve a DOD jetting process, a pulse current is generated from a direct power source via a controller and applied on the liquid metal, and a Lorentz force is instantaneously induced, by which the liquid metal is ejected from the nozzle to form micro droplets, as shown in Figure 1. In this case, the duration (the sum of the rising and falling edge time of pulse current) of the instantaneous Lorentz force on the liquid can be minimized to the microsecond ( $\mu$ s) scale, which can break through the bottleneck of the low jetting frequency currently encountered by other techniques. Moreover, EMJ allows further structural simplification so that the stability of the system can be improved. Experimentally, the following parameters are used: a magnetic field intensity of $0.4 \mathrm{~T}$, a current of approximately $30 \mathrm{~A}$, a voltage of $12 \mathrm{~V}$, a pulse width of $0.5 \mathrm{~ms}$, a distance between electrodes of $36 \mathrm{~mm}$, and an outlet diameter of about $1 \mathrm{~mm}$. According to its working principle, theoretically, EMJ can be used for micro-jetting any kind of liquid metal; however, a high temperature weakens magnetic fields, and more particularly, permanent magnets have their Curie-temperatures. Therefore, the temperature of the liquid metal should not be too high, or a cooling device should be provided to prevent the magnet from overheating. Currently, preferable EMJ metallic materials include gallium, mercury, and a soldering tin alloy. Metallic gallium $(\mathrm{Ga})$ was selected for the experiment because of its low melting point $\left(\sim 29.8^{\circ} \mathrm{C}\right)$ and good stability in the air. With this low melting point, 3D printing of gallium can be realized by micro-jetting, which is a direct $3 \mathrm{D}$ printing of metallic parts.

A $3 \mathrm{D}$ view and parameters of the experimental device is shown in Figure $2 \mathrm{a}$, a 2D view shown in Figure $2 b$, and schematics of the jetting valve shown in Figure 2c. The jetting performance of liquid Ga was evaluated by varying the lead angle of the nozzle from $60^{\circ}$ to $120^{\circ}$, as shown in Figure $3 a$. It should be noted that the data are the average of 10 samplings of each experiment, and the jet is considered to follow the horizontal projectile motion at the very beginning. According to Newton's second law and kinetic energy theorem, Equations (1) and (2) can be deduced, based on which key parameters including the initial velocity $\left(V_{0}\right)$ and the kinetic energy of the droplet $\left(E_{\mathrm{k}}\right)$ can be calculated, whereas the jetting distance $\left(S_{1}\right)$ and the mass $(m)$ of the jet droplets can be measured in the experiment.

$$
V_{0}=S_{1} \times \sqrt{\frac{g}{2 h}}
$$




$$
E_{\mathrm{k}}=\frac{1}{2} m V_{0}^{2}=\frac{m g S_{1}^{2}}{4 h}
$$

where $g$ is gravity acceleration; $h$ is the nozzle height $(h=23.75 \mathrm{~mm})$.

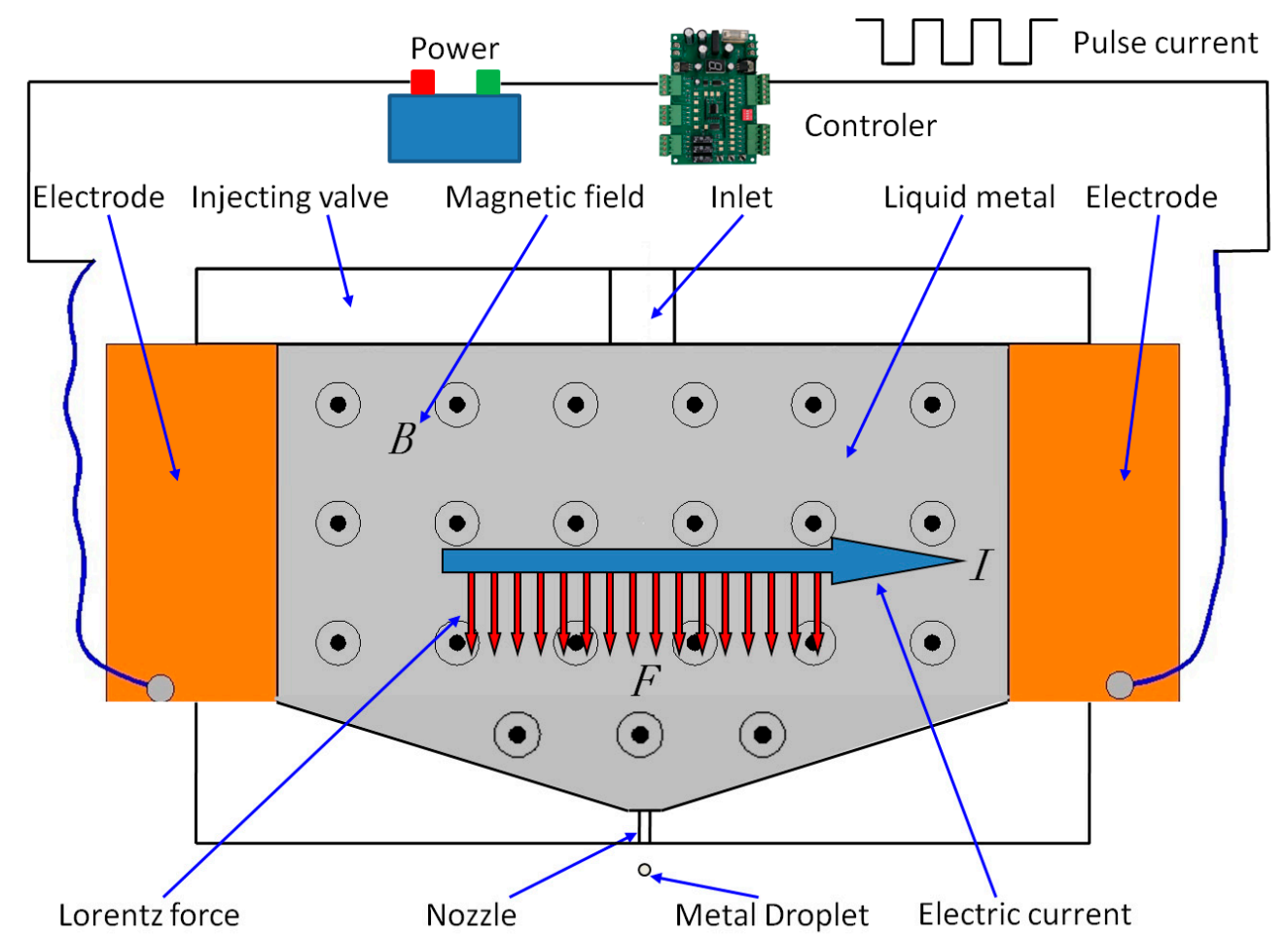

Figure 1. Schematic illustration of electromagnetic micro-jetting (EMJ).

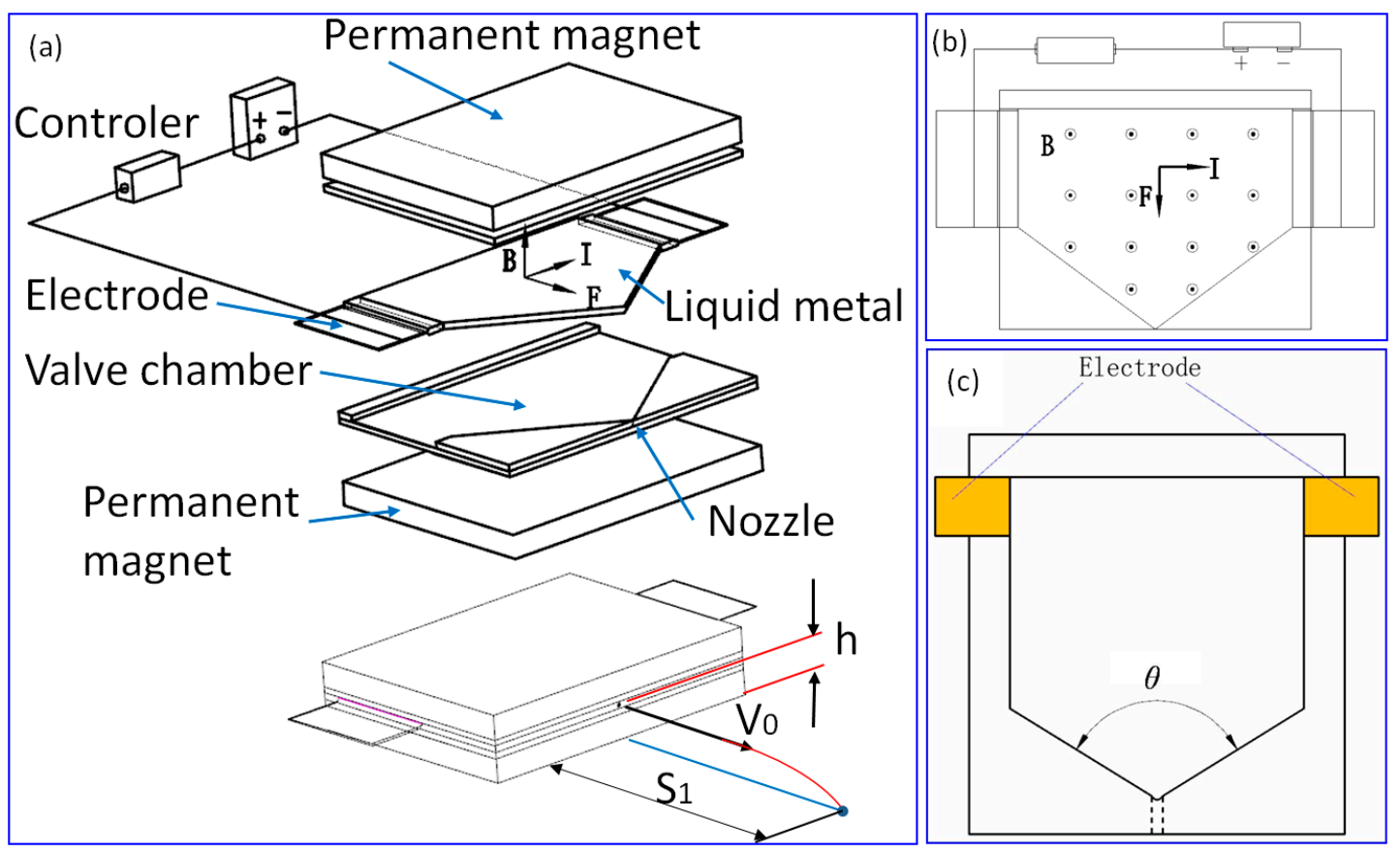

Figure 2. (a) 3D view and parameters of the experiment device. (b) 2D view of the experiment device. (c) Schematics of the jetting valve. 

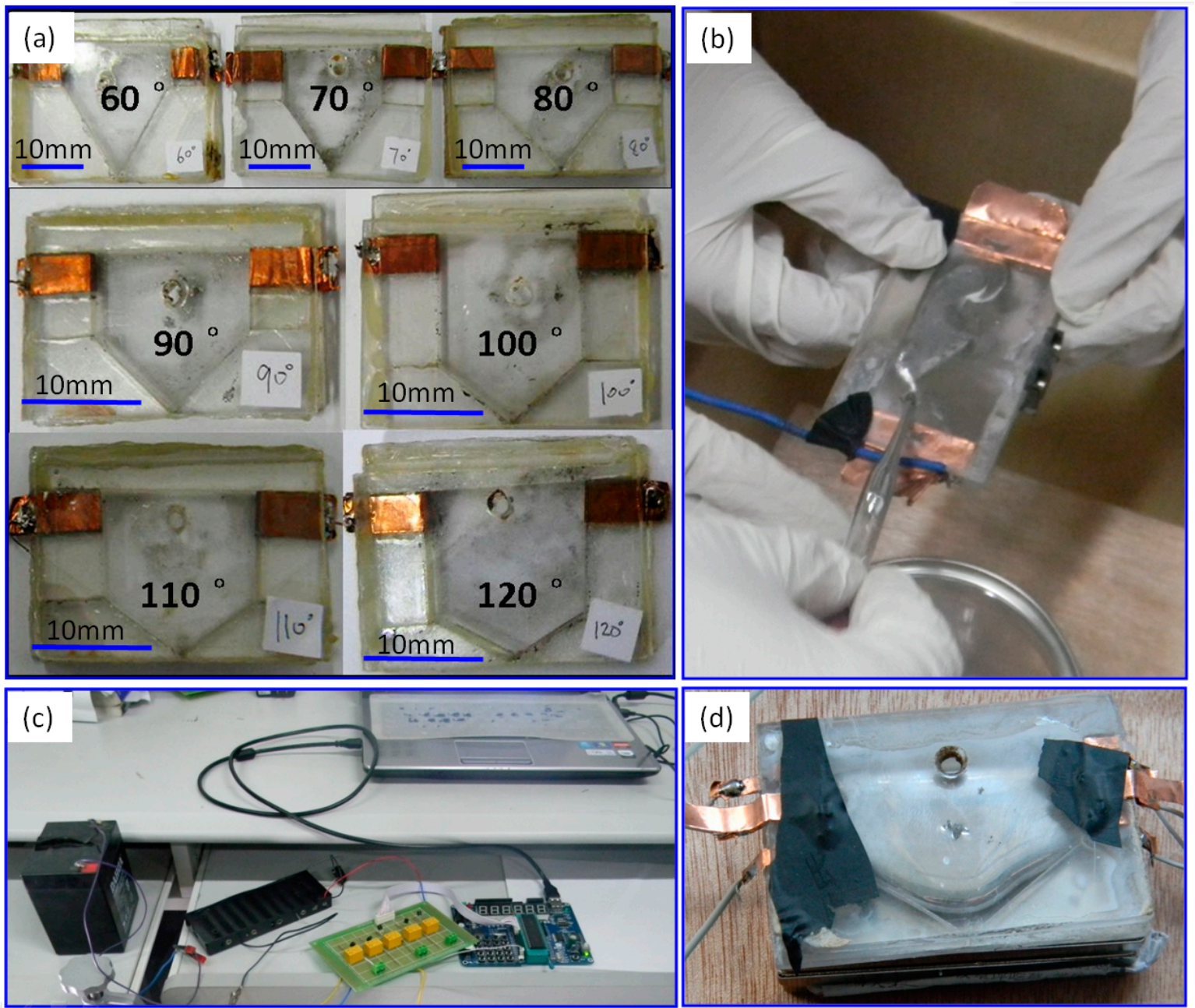

Figure 3. (a) Fabricated prototypes of jetting valves with different lead angles ( $\theta$ ). (b) Filling gallium into the valve with a dropper. (c) The controller and the power source. (d) The jetting valve loaded with gallium.

The droplet jetting motion, here, can be taken as the parabolic motion with initial velocity $V_{0}$ in the horizontal direction; then, the jetting motion can be derived from Equations (1) and (2).

In order to observe the liquid motion inside the jetting valve, while ensuring current flows through the liquid metal rather through the valve body, clear acrylic boards are used to fabricate the jetting valve. The electrode is fabricated with copper foil so that the current can flow from outside the valve body to the metal liquid inside. All components of the jetting valve are bonded together as a whole with super glue to ensure sealing tightness, as shown in Figure 3a. In the design of this jetting valve, the inlet is located on top of the wide panel, through which gallium is filled in with a dropper, as shown in Figure 3b. The current controller uses an Microcontroller Unit MCU-based (SST89E564RD) control circuit to generate a pulse current with a modulated pulse width, frequency, duty ratio, etc.; the control circuit is isolated from the working current with a relay (HK4100F-DC5V-SHG, HuiKe relay Co., Ltd, Shenzhen, China). A battery UPS 12V6AH (Panasonic Storage Battery(Shenyang) Co., Ltd, Shenyang, China) is used as the power source as shown in Figure 3c. The current of the working circuit is above $30 \mathrm{~A}$ due to small loads in the loop. The jetting valve loaded with gallium is shown in Figure $3 \mathrm{~d}$. Neodymium magnets are used to provide the valve with a stable magnetic field, with an intensity up to $0.4 \mathrm{~T}$. 


\section{Results and Discussion}

The jetting valve in working status is shown in Figure $4 \mathrm{a}$, and an enlarged view of the round-shaped nozzle is shown in Figure $4 \mathrm{~b}$, with a diameter of $686 \mu \mathrm{m}$. In the experiment, the jetting valve is positioned horizontally, and a round plastic disc is placed in front of it as the collector. The jetting distance $S_{1}$ is measured with a steel ruler, as shown in Figure 4c. Figure 4d shows metal droplets collected in the disc. A top view of the EMJ process is shown in Figure 4e, and a side view shown in Figure 4f. During the experiment, sometimes the magnet on top was removed to observe the motion of the metal liquid inside the jetting valve, in which circumstance the valve was still functioning. However, all experimental data were collected when both magnets on the top and bottom were installed. The weight of the droplets was measured with an analytical balance. The plastic disc was used as the collecting panel at the beginning, and was then replaced with sticky tapes to prevent droplets from moving around. The experiment was carried out in summer, with a room temperature of $30{ }^{\circ} \mathrm{C}$ and a humidity of $50 \%$.

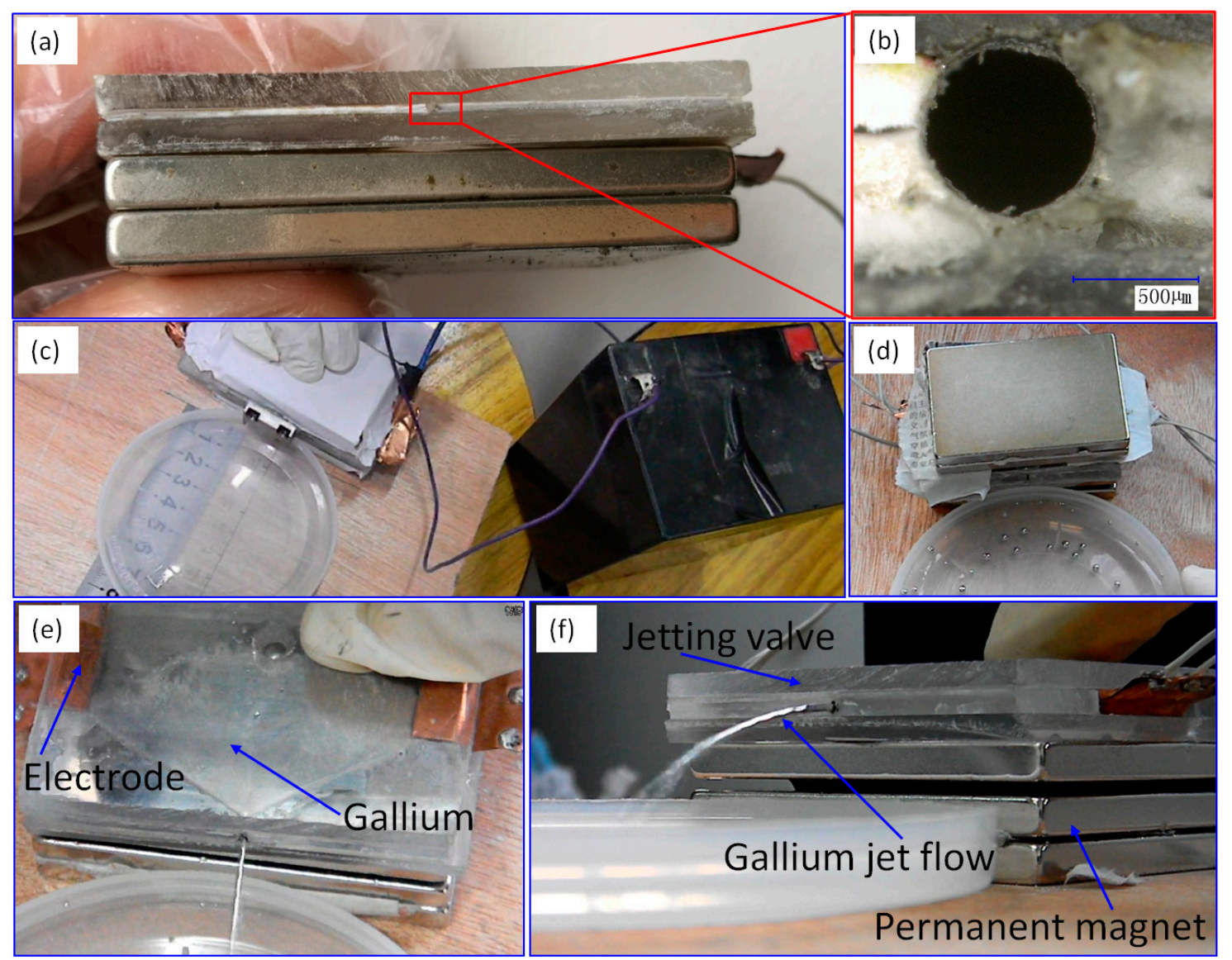

Figure 4. (a) The jetting valve. (b) The nozzle. (c) The experiment platform. (d) Droplets collection. (e) A top view of the jetting process. (f) A side view of the EMJ process.

As the lead angle of the nozzle changes, the mass of liquid Ga in the cavity (between the nozzle and electrodes) changes accordingly, so does the Lorentz force component parallel to the nozzle. Figure 5 shows the mass of Ga droplets deposited under different lead angles. It is clear that the mass of droplets increases with the increase in the lead angle from $60^{\circ}$ to $90^{\circ}$, but decreases when further increasing the angle $\left(90^{\circ}<\theta \leq 120^{\circ}\right)$. This trend results from the competition between the Lorentz force component and the internal resistance of liquid $\mathrm{Ga}$ in the cavity. The resistance of the liquid $\mathrm{Ga}$ increases as the angle increases from $60^{\circ}$ to $120^{\circ}$, while the Lorentz force component (parallel to the nozzle) only increases along with the angle from $60^{\circ}$ to $90^{\circ}$, and then decreases from $90^{\circ}$ to $120^{\circ}$. 
From $60^{\circ}$ to $90^{\circ}$, the Lorentz force component (parallel to the nozzle) exhibits a much larger increase than the resistance of the liquid Ga. Therefore, a greater volume of the droplet is ejected with larger angles, and EMJ at the angle of $90^{\circ}$ produces the largest and heaviest droplets (see inset on top).

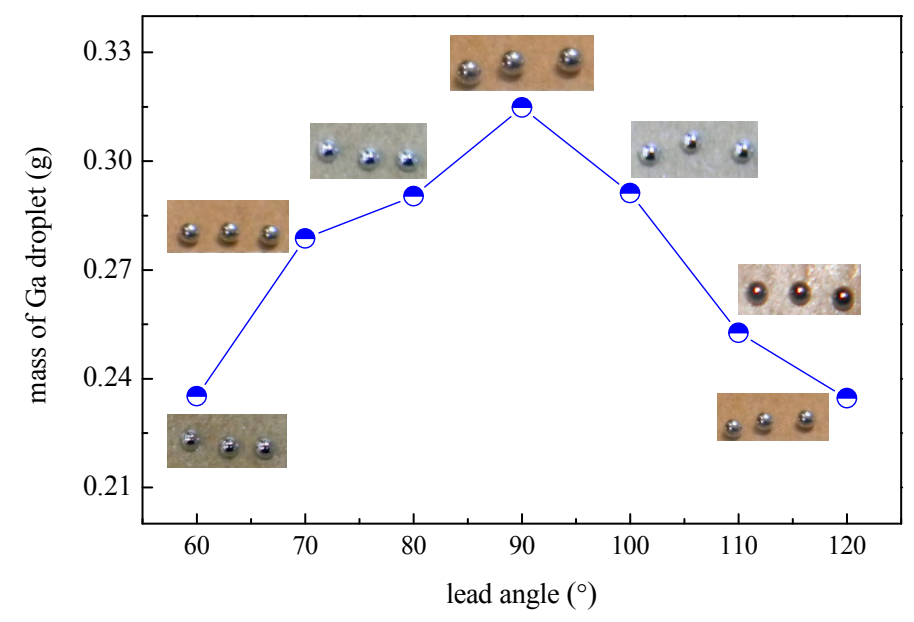

Figure 5. Mass of liquid Ga droplets as a function of the lead angle.

It is well known that the jetting distance $\left(S_{1}\right)$ is closely correlated with the initial velocity of the jet in a horizontal projectile motion. As mentioned above, $S_{1}$ can be directly measured, which shows an increase with the angle growing from $60^{\circ}$ to $80^{\circ}$, and then decreases afterwards (figure not shown here). According to Equation (1), the initial velocity $\left(V_{0}\right)$ should have a very similar profile to $S_{1}$, as plotted in Figure $6 \mathrm{a}$, where a maximum $V_{0}$ of $0.042 \mathrm{~m} / \mathrm{s}$ is calculated for $80^{\circ}$. Although the Lorentz force component (parallel to the nozzle) achieves the maximum at $90^{\circ}$, the mass of droplets $(m)$ also reaches its maximum, resulting in the increased internal friction of the liquid, which could be the reason for a lower $V_{0}$ at $90^{\circ}$ than at $80^{\circ}$. According to Equation (2), the kinetic energy of the droplet $\left(E_{\mathrm{k}}\right)$ can be further determined, as plotted in Figure $6 \mathrm{~b}$. Since $E_{\mathrm{k}}$ is proportional to $\mathrm{m}$ (the mass of droplet) and the square of $V_{0}$ (initial velocity of the jet), the profile of $E_{\mathrm{k}}$ is more affected by $V_{0}$. Hence, very close and comparatively high values of $E_{\mathrm{k}}$ are obtained at $80^{\circ}$ and $90^{\circ}$, suggesting that these two angles, and that in between, can provide a preferable performance. In addition, the size of the nozzle has a great influence on the diameter of the collected droplets. In the initial design, the nozzle diameter was relatively large $(600 \mu \mathrm{m})$, which generates droplets with diameters up to $3 \mathrm{~mm}$. Later, a stainless steel nozzle that produces droplets with a diameter of about $350 \mu \mathrm{m}$ was fabricated (with a diameter of $250 \mu \mathrm{m})$.

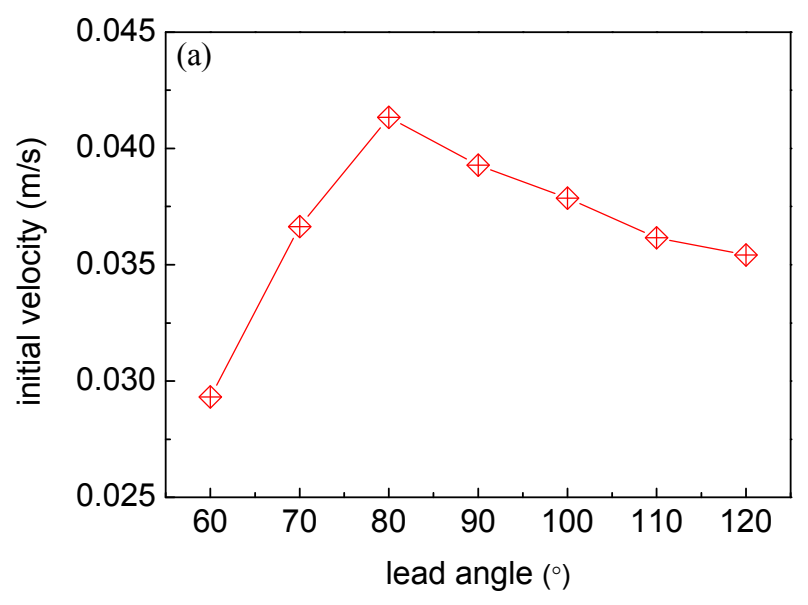

Figure 6. Cont. 


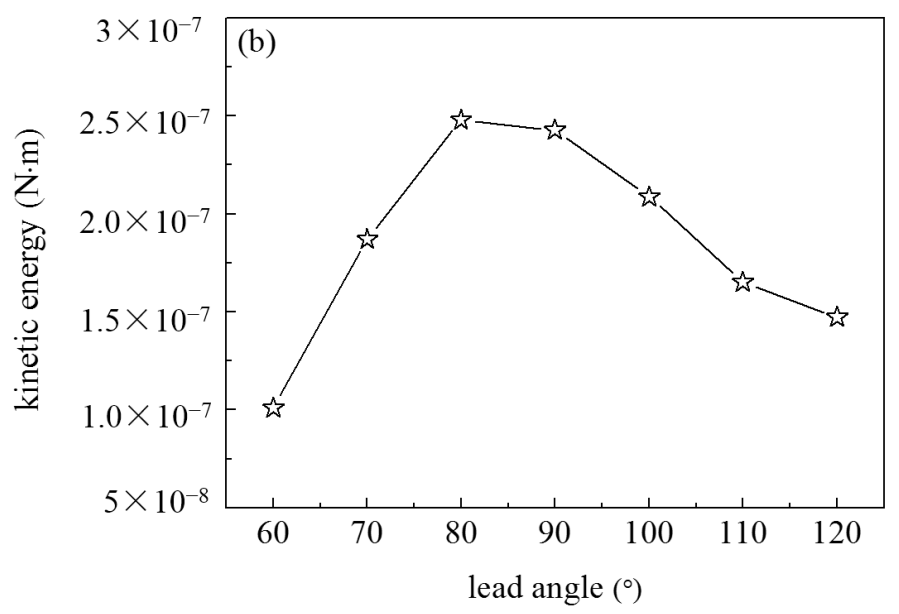

Figure 6. Initial velocity of the jet (a) and kinetic energy of the droplet (b) as a function of the lead angle respectively.

From the above results, it is reasonable to state that the lead angle has a great influence on jetting performance and the resultant droplets. The production of regular liquid Ga droplets on a micro-scale size preliminarily demonstrates the feasibility of using the EMJ technique to deposit liquid metal in a DOD manner, which we believe has great potential in various manufacturing industries.

\section{Conclusions}

Based on the Lorentz force induced by charges moving in a magnetic field, an EMJ valve capable of drop-on-demand (DOD) jetting is here proposed, and the influence of the nozzle lead angle on the jetting of gallium (Ga) was investigated. Results show that jetting processes, with different initial velocities, occur under all lead angles, because the component of the Lorentz force parallel to the nozzle for the electrified liquid $\mathrm{Ga}$ is always greater than its internal friction. The mass of the jet liquid, the jetting distance, the initial velocity of the jet, and the resulting kinetic energy of the jet are found to increase first and then decrease. When the lead angle is $90^{\circ}$, the mass and the kinetic energy are at their maximum, while the initial velocity achieves maximum at $80^{\circ}$. Very close and comparatively high kinetic energies are obtained at $80^{\circ}$ and $90^{\circ}$, indicating that a preferable performance can be achieved with angles in between this range. Future improvement on its design allows for better jetting performance of the EMJ technique, which we believe has great potentials in flexible printed circuits, large-scale integrated circuit board welding, and so on.

Acknowledgments: This work was financially supported by the Natural Science Foundation of Fujian Province of China (2015J01227), the Natural Science Foundation of China (No. 51575464), Xiamen Science and Technology Plan Projects (3502Z20153028), and the Fujian Educational Committee Foundation (JA13237).

Author Contributions: All work with relation to this paper was accomplished by all of the authors' efforts. The idea and the design of the liquid metal electromagnetic micro-jetting were proposed by Zhiwei Luo. Gaofeng Zheng designed the fabrication method of the jetting dispenser. Jetting experiments were completed with the help of Lingyun Wang, who also provided significant guidance on the selection of the experiment method. At last, every segment related to this paper is accomplished under the guidance from Gaofeng Zheng.

Conflicts of Interest: The authors declare no conflict of interest.

\section{References}

1. Orme, M. A novel technique of rapid solidification net-form materials synthesis. J. Mater. Eng. Perform. 1993, 2, 399-405. [CrossRef]

2. Xu, L.; Wang, X.; Lei, T.; Sun, D.; Lin, L. Electrohydrodynamic deposition of polymeric droplets under low-frequency pulsation. Langmuir 2011, 27, 6541-6548. [CrossRef] [PubMed] 
3. Huang, H.; Qi, L.; Zeng, X.; Yang, F. Principle of micro-feeding powders by ultrasonic vibration and influence of amplitude on rate of micro-feeding powders. Chin. J. Mech. Eng. 2009, 1, 267-272. [CrossRef]

4. Park, B.K.; Kim, D.; Jeong, S.; Moon, J.; Kim, J.S. Direct writing of copper conductive patterns by ink-jet printing. Thin Solid Films 2007, 515, 7706-7711. [CrossRef]

5. Šturm, R.; Grum, J.; Božič, S. Influence of the alloying elements in Al-Si alloys on the laser remelting process. Lasers Eng. (Old City Publ.) 2012, 22, 47-61.

6. Yokoyama, Y.; Endo, K.; Iwasaki, T.; Fukumoto, H. Variable-size solder droplets by a molten-solder ejection method. J. Microelectromech. Syst. 2009, 18, 316-321. [CrossRef]

7. Song, Q.; Zhu, W.; Zhang, W.; Ren, M.; Chia, E.; Liu, A. Tunable thz filter based on random access metamaterial with liquid metal droplets. In Proceedings of the 2014 IEEE 27th International Conference on Micro Electro Mechanical Systems (MEMS), San Francisco, CA, USA, 26-30 January 2014; pp. 664-667.

8. Sun, J.; Fuh, J.; Thian, E.; Hong, G.; Wong, Y.; Yang, R.; Tan, K. Fabrication of electronic devices with multi-material drop-on-demand dispensing system. Int. J. Comput. Integr. Manuf. 2013, 26, 897-906. [CrossRef]

9. Glynne-Jones, P.; Coletti, M.; White, N.M.; Gabriel, S.; Bramanti, C. A feasibility study on using inkjet technology, micropumps, and MEMS as fuel injectors for bipropellant rocket engines. Acta Astronaut. 2010, 67, 194-203. [CrossRef]

10. Kim, B.-H.; Kim, S.-I.; Lee, J.-C.; Shin, S.-J.; Kim, S.-J. Dynamic characteristics of a piezoelectric driven inkjet printhead fabricated using MEMS technology. Sens. Actuators A Phys. 2012, 173, 244-253. [CrossRef]

11. Vaezi, M.; Chua, C.K. Effects of layer thickness and binder saturation level parameters on 3D printing process. Int. J. Adv. Manuf. Technol. 2011, 53, 275-284. [CrossRef]

12. Lijun, L.J.L. Experimental study on fabrication of thin-wall metallic features by laser cladding. Chin. J. Mech. Eng. 2004, 10, 036.

13. Xiao, Y.; Qi, L.; Huang, H.; Zeng, X.-H.; Yang, F. Design and implement of pneumatic drop-on-demand generator for metal droplet production. Trans. Beijing Inst. Technol. 2010, 30, 780-784.

14. Zeng, X.; Qi, L.; Huang, H.; Li, Y.; Yang, F. Control system development and experimental study on ultrasonic vibration feeding. Chin. J. Mech. Eng. 2011, 24, 1083-1088. [CrossRef]

15. Cheng, S.X.; Li, T.; Chandra, S. Producing molten metal droplets with a pneumatic droplet-on-demand generator. J. Mater. Process. Technol. 2005, 159, 295-302. [CrossRef]

16. Cao, W.; Miyamoto, Y. Freeform fabrication of aluminum parts by direct deposition of molten aluminum. J. Mater. Process. Technol. 2006, 173, 209-212. [CrossRef]

17. Chun, J.-H.; Passow, C.H. Production of Charged Uniformly Sized Metal Droplets. U.S. Patent 5266098, 30 November 1993.

18. Orme-Marmerelis, M.; Smith, R.F. High-Speed Fabrication of Highly Uniform Metallic Microspheres. U.S. Patent 6562099, 18 May 2003.

(C) 2016 by the authors; licensee MDPI, Basel, Switzerland. This article is an open access article distributed under the terms and conditions of the Creative Commons Attribution (CC-BY) license (http://creativecommons.org/licenses/by/4.0/). 